\title{
Maturation Phenotype of Peripheral Blood Monocyte/Macrophage After Stimulation with Lipopolysaccharides in Irritable Bowel Syndrome
}

\author{
Oscar A Rodríguez-Fandiño, ${ }^{1,2}$ Joselín Hernández-Ruiz, ${ }^{1}$ Yolanda López-Vidal, ${ }^{3}$ Luis Charúa-Guindic, ${ }^{4}$ Galileo Escobedo, ${ }^{1}$ and \\ Max J Schmulson ${ }^{1 *}$ \\ ${ }^{1}$ Laboratorio de Hígado, Páncreas y Motilidad (HIPAM), Unidad de Investigación en Medicina Experimental, Facultad de Medicina-Universidad \\ Nacional Autónoma de México (UNAM), Hospital General de México, Dr. Eduardo Liceaga, Mexico City, Mexico; ${ }^{2}$ Dirección de Investigación, \\ Fundación Universitaria-Unitrópico, Yopal, Colombia; ${ }^{3}$ Programa de Inmunología Molecular Microbiana, Departamento de Microbiología y \\ Parasitología, Facultad de Medicina-Universidad Nacional Autónoma de México (UNAM), Mexico City, Mexico; and ${ }^{4}$ Unidad de Coloproctología, \\ Hospital General de México, Dr. Eduardo Liceaga, Mexico City, Mexico
}

\section{Background/Aims}

Abnormal immune regulation and increased intestinal permeability augmenting the passage of bacterial molecules that can activate immune cells, such as monocytes/macrophages, have been reported in irritable bowel syndrome (IBS). The aim was to compare the maturation phenotype of monocytes/macrophages (CD14+) from IBS patients and controls in the presence or absence of Escherichia coli lipopolysaccharides (LPS), in vitro.

\section{Methods}

Mononuclear cells were isolated from peripheral blood of 20 Rome II-IBS patients and 19 controls and cultured with or without LPS for 72 hours. The maturation phenotype was examined by flow cytometry as follows: M1-Early (CD11 $\left.C^{+} C D 206\right)$, M2-Advanced $\left(\mathrm{CD} 11 \mathrm{C}^{-} \mathrm{CD} 206^{+} \mathrm{CX} 3 \mathrm{CR} 1^{+}\right)$; expression of membrane markers was reported as mean fluorescence intensity (MFI). The Mann-Whitney test was used and significance was set at $P<0.05$.

\section{Results}

In CD14+ cells, CD11c expression decreased with vs without LPS both in IBS (MFI: $8766.0 \pm 730.2$ vs $12920.0 \pm 949.2, P<0.001$ ) and controls (8233.0 \pm 613.9 vs $13750.0 \pm 743.3, P<0.001)$. M1-Early cells without LPS, showed lower CD11c expression in IBS than controls (MFl: $11540.0 \pm 537.5$ vs $13860.0 \pm 893.7, P=0.040$ ), while both groups showed less CD11c in response to LPS ( $P$ $<0.01)$. Furthermore, the percentage of "Intermediate" $\left(C D 11 C^{+} C D 206^{+} C X 3 C R 1^{+}\right)$cells without LPS, was higher in IBS than controls (IBS $=9.5 \pm 1.5 \%$ vs $C=4.9 \pm 1.4 \%, P<0.001)$. Finally, fractalkine receptor (CX3CR1) expression on M2-Advanced cells was increased when treated with LPS in controls but not in IBS $(P<0.001)$

\section{Conclusions}

The initial phase of monocyte/macrophage maturation appears to be more advanced in IBS compared to controls. However, the decreased CX3CR1 in patients with IBS, compared to controls, when stimulated with LPS suggests a state of immune activation in IBS.

(J Neurogastroenterol Motil 2017;23:281-288)

\section{Key Words}

Fractalkine receptor; Irritable bowel syndrome; Lipopolysaccharides; Monocytes

Received: August 24, 2016 Revised: None Accepted: October 26, 2016

(a) This is an Open Access article distributed under the terms of the Creative Commons Attribution Non-Commercial License (http://creativecommons. org/licenses/by-nc/4.0) which permits unrestricted non-commercial use, distribution, and reproduction in any medium, provided the original work is properly cited.

*Correspondence: Max J Schmulson, MD

Laboratorio de Hígado, Páncreas y Motilidad (HIPAM), Unidad de Investigación en Medicina Experimental, Facultad de Medicina, Universidad Nacional Autónoma de México (UNAM), Hospital General de México, Dr. Eduardo Liceaga, Dr. Balmis \#148. Col. Doctores, C.P. 06726, México D.F., México

Tel: +52-5556232673, Fax: +52-5556232669, E-mail: maxjulio@prodigy.net.mx 


\section{Introduction}

Despite the high prevalence of functional gastrointestinal disorders, as is the case with irritable bowel syndrome (IBS), the underlying biologic mechanisms are not completely understood. Nevertheless, the recognition of post-infectious IBS (PI-IBS) ${ }^{1}$ and the risk for presenting IBS after antibiotic use, ${ }^{2}$ suggest an association of this disorder with the presence of an altered microbiota/ microbiome and the consequent generation or exacerbation of IBS symptomatology. This knowledge brought about a change of focus in the search for the factors involved in IBS, directing attention to the different microbial species living in the intestine ${ }^{3}$ and their relation to the immune cells recognition and response in terms of cytokines or chemokines. ${ }^{4,5}$ As a consequence, numerous efforts have been made to understand the participation of: (1) the types of immune response (innate and acquired), the state of inflammation, and immune regulation; ${ }^{6}(2)$ the behavior of the pathogenic and commensal bacterial communities, ${ }^{7}$ their response and symptomatology after the use of antibiotics ${ }^{8}$ and probiotics; ${ }^{9,10}$ and (3) the alterations in intestinal epithelium permeability ${ }^{11,12}$ in the genesis of IBS. The immunologic hypothesis inferred in relation to IBS is that of a transformation to a presumed state of low-grade inflammation and/or immune activation. For example, there is an imbalance in pro- and anti-inflammatory cytokines in IBS which is subtype and sex dependent. ${ }^{13}$ Even though none of the proposals is completely accepted, evidence has shown that at least one subgroup of patients can present with these alterations. ${ }^{13}$

Thus, it is necessary to examine the response of the cell groups involved in immune regulation in IBS, which includes the monocytes/macrophages population. ${ }^{14}$ The maturation process of monocytes into macrophages that takes place in the intestine is carried out by a continuous flow of monocytes coming from the blood stream. ${ }^{15}$ This monocyte recruitment provides a constant intestinal replenishment of macrophage populations that gradually mature in a cascade-like manner until they reach the level of resident macrophages. ${ }^{16,17}$ Our hypothesis was that patients with IBS may show an alteration in the maturation cascade of monocytes into macrophages in response to bacterial components in charge of triggering this disorder. Therefore our aim was to analyze the maturation phenotype of monocytes/macrophages (CD14+) from the peripheral blood of patients with IBS and controls, by in vitro exposing CD14+ cells to Escherichia coli-derived lipopolysaccharides (LPS).

\section{Materials and Methods}

\section{Study Population}

Patients between 18 to 60 years of age that have previously been evaluated at our laboratory, ${ }^{18}$ and consulted at the colorectal clinic of the Hospital General de México, Dr. Eduardo Liceaga, within the time frame of May to October 2011, were studied. This hospital is a tertiary care center and is considered the most prominent within the public health system in Mexico. Independently of the reason for consultation, all patients seen at the colorectal clinic routinely undergo rigid rectosigmoidoscopy. Those patients that showed no endoscopic evidence of any organic disease were eligible to participate in our study. Also, their medical history had to be negative for inflammatory bowel disease (IBD), celiac disease, microscopic colitis, or gastrointestinal cancer. Likewise, those patients with current signs or symptoms of gastrointestinal or respiratory infectious diseases, those using proton pump inhibitors, antispasmodics, prokinetics, antibiotics, antidepressants, or anti-inflammatory or immunomodulatory agents were excluded from the study. The patients that met the above criteria and voluntarily accepted to participate in the study signed informed consent for their inclusion.

\section{Rome II Modular Questionnaire}

All the subjects fulfilling the above criteria answered the Rome II Modular Questionnaire validated in Mexico ${ }^{19,20}$ to further categorize them as IBS or controls, regardless of the reason for their medical consultation. IBS was considered when there was abdominal pain or discomfort for at least 12 weeks in the last 12 months that was associated with improvement after defecation and/or associated with a change in defecation frequency and/or associated with a change in stool consistency. ${ }^{21}$ The patients with IBS were then classified as diarrhea-predominant IBS (IBS-D) or constipationpredominant IBS (IBS-C), whereas the individuals that did not fit the IBS-D or IBS-C characteristics were classified as alternating/ mixed IBS (IBS-A/M). In addition, IBS diagnosis was confirmed by a gastroenterologist with experience in IBS (M.S.). Furthermore, none of the patients had a history of gastroenteritis as a triggering factor of IBS (in other words, PI-IBS). ${ }^{22}$ The controls were subjects that did not meet criteria for IBS or any other functional gastrointestinal disorder according to the Rome II Modular Questionnaire, and those that had no history of any chronic gastrointestinal disorders as previously described (Study Population), or any other chronic pain syndrome. 


\section{Isolation of the Mononuclear Cells}

Peripheral venous blood samples were collected from each of the study subjects and were packed in sterile EDTA vacutainers (BD Biosciences, San Jose, CA, USA). The mononuclear cells (MNCs) were immediately isolated for centrifugation at the concentration gradient. Fifteen milliliters of blood were diluted in a filtered phosphate-buffered saline solution (PBS) (volume 1:1) and a layer was carefully added to $15 \mathrm{~mL}$ of Histopaque-1077 (Sigma, St Louis-MO, USA) in a $50 \mathrm{~mL}$ sterile centrifugation tube and centrifuged at $400 \mathrm{~g}$ for 20 minutes at room temperature. The MNCs were washed, once with lysis buffer and twice with PBS, to finally evaluate their viability through the trypan blue exclusion test. The MNCs were resuspended at a volume of $2 \times 10^{6}$ cells $/ \mathrm{mL}$ in complete medium (RPMI 1640; Gibco, Grand Island, NY, USA), supplemented with $10 \%$ fetal bovine serum (Gibco), $100 \mathrm{IU} / \mathrm{mL}$ penicillin, and $10 \mathrm{mg} / \mathrm{mL}$ streptomycin (Gibco). ${ }^{23}$

\section{Cell Cultures}

The MNCs were cultured in 24-well plaques (Costar, Corning Inc, Wilkes Barre, PA, USA) with no stimulus or with $50 \mathrm{mg} / \mathrm{mL}$ of LPS from E. coli (Sigma-Aldrich, St. Louis, MO, USA). The reason for using that particular ligand was the association that has been reported between toll-like receptor 4 stimulation and IBS. ${ }^{18,24}$ The cell cultures were incubated for 72 hours at $35^{\circ} \mathrm{C}, 5 \% \mathrm{CO}_{2}$, and a humidified atmosphere. Finally, the resulting cells were preserved in dimethyl sulfoxide $10 \%$ at $-80^{\circ} \mathrm{C}$ until measuring time.

\section{Flow Cytometry}

The MNCs preserved at $-80^{\circ} \mathrm{C}$ were thawed using the modified Germann et $\mathrm{al}^{25}$ technique. In short, the cells were rapidly thawed in a water bath $\left(37^{\circ} \mathrm{C}\right)$, diluted in PBS (volume 1:10), centrifuged at $400 \mathrm{~g}$ for 5 minutes, and resuspended in $50 \mu \mathrm{L}$ of PBS. ${ }^{25}$ Briefly, as the monocytes/macrophages (CD14+ cells were used for this study) mature, they differentially express a series of membrane receptors, measured in terms of mean fluorescence intensity (MFI), as is the case with CD11c, CD206, and the fractalkine receptor (CX3CR1). These receptors let us examine the degree of development within the maturation cascade. In the most immature state, the cells are negative for the 3 markers. CD11c is more rapidly expressed $\left(\mathrm{CD} 11 \mathrm{c}^{+} \mathrm{CD} 206^{-}\right.$, conventionally known as $\mathrm{M} 1$, which under the cascade proposal is more an early state) and as the cell matures, the receptor again declines. CD206 and CX3CR1 are slowly expressed as the cells continue to develop (CD11. CD206 ${ }^{+}$, conventionally known as M2), which under the proposal of cascade maturation corresponds to a more advanced state, the same as CX3CR1, whose expression is related to mature phenotypes, mainly immune regulators. ${ }^{17,26}$ The proposed maturation cascade is depicted in Figure 1.

\section{Statistical Analysis}

The continuous variables were expressed as mean $\pm \mathrm{SD}$. The data were analyzed using the non-parametric Mann-Whitney test. Statistical significance was set at a value of $P<0.05$. The SPSS version 17.0 (SPSS, Chicago, IL, USA) statistical program was employed. The study protocol was approved by the Ethics and Research Committee of the Hospital General de México, Dr. Eduardo Liceaga. In addition, a post hoc analysis for Mann-Whitney test ( $G$ Power 3.1.9.2; Institut für Experimentelle Psychologie, Christian-Albrechts-Universität Kiel, Kiel, Germany) was conducted to evaluate the statistical power of the obtained results between the IBS and controls. The power was higher than 0.94 for the statistically significant differences.

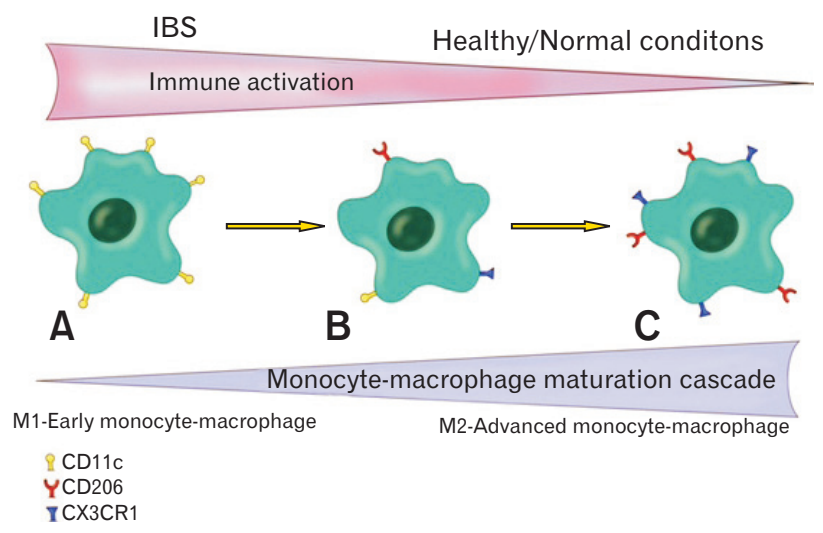

Figure 1. Schematic representation of the proposed maturation cascade of monocytes/macrophages. In normal conditions where no immune activation is seen, monocytes-macrophages exhibit a M1Early state of maturation characterized by increased CD11c expression $\left(\mathrm{CD} 11 \mathrm{c}^{+} \mathrm{CD} 206^{-}\right)$. As immune activation raises, monocytesmacrophages progressively lose CD11c expression and show moderate expression of both CD206 and CX3CR1, thus constituting an intermediate maturation phenotype $\left(\mathrm{CD} 11 \mathrm{c}^{+} \mathrm{CD} 206^{+} \mathrm{CX} 3 \mathrm{CR} 1^{+}\right)$(lower part in gray). In IBS patients, monocytes-macrophages display a more activated basal state based on a lower expression of CD11c in M1 cells in cultures without LPS stimulation. However an altered immune regulatory state is observed in IBS suggested by a lower expression of CX3CR1 (upper part in red). A, M1-early maturation phenotype; B, intermediate maturation phenotype; and $\mathrm{C}, \mathrm{M} 2$-advanced maturation phenotype. 


\section{Results}

The Table describes the general characteristics of the study groups. Age and sex distribution were similar between the IBS patients and the controls. Almost half (45\%) of the patients with IBS were classified as IBS-A/M, followed by IBS-C (40\%), and IBS-D $(15 \%)$.

\section{Monocyte/Macrophage Phenotype}

Based on the previously described maturation cascade, in CD14+ cells cultured with LPS compared with those in cultures with no LPS stimulation, we found a lower expression of CD11c. These findings were observed in the IBS patients $(8766.0 \pm 730.2$ vs $12920.0 \pm 949.2, P<0.001)$, as well as for the controls (8233.0 \pm 613.9 vs $13750.0 \pm 743.1, P<0.001)$, with no statistically significant differences between the groups (IBS vs Controls) (Fig. 2). Based on CD11c and CD206 expression, the subgroups were

Table. Demographic Characteristics of the Study Groups

\begin{tabular}{lccc}
\hline & $\begin{array}{c}\text { IBS } \\
\mathrm{n}=20\end{array}$ & $\begin{array}{c}\text { Controls } \\
\mathrm{n}=19\end{array}$ & P-value \\
\hline Sex $(\mathrm{W} / \mathrm{M})$ & $15 / 5$ & $13 / 6$ & 0.580 \\
Age (mean $\pm \mathrm{SD}, \mathrm{yr})$ & $41.9 \pm 12.3$ & $40.7 \pm 9.8$ & 0.730 \\
IBS subtype (C/D/A) & $8 / 3 / 9$ & - & - \\
\hline
\end{tabular}

IBS, irritable bowel syndrome; W, women; M, men; C, constipation; D, diarrhea; A, alternating/mixed.

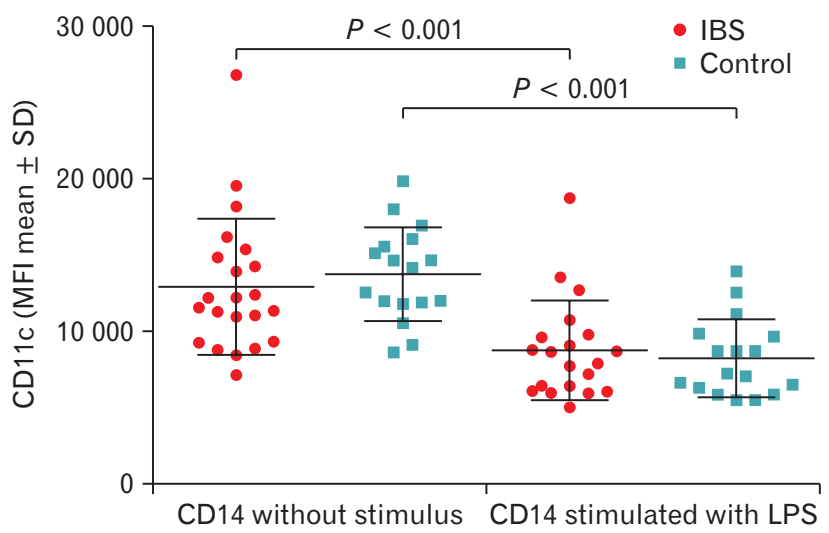

Figure 2. CD11c expression in CD14+ monocytes, in cultures with and without lipopolysaccharide (LPS) stimulation from irritable bowel syndrome (IBS) patients and controls. The mononuclear cell $(\mathrm{CD} 14+)$ culture stimulated with LPS shows a decrease in membrane receptor (CD11c) mean fluorescence intensity (MFI) expression in patients with IBS and in controls. mainly composed of $\mathrm{CD} 11 \mathrm{c}^{+} \mathrm{CD} 206^{+}$(intermediate state), followed by $\mathrm{CD} 11 \mathrm{c}^{-} \mathrm{CD} 206^{+}$(advanced state), and a smaller proportion of $\mathrm{CD} 11 \mathrm{c}^{+} \mathrm{CD} 206$ (early state).

In $\mathrm{M} 1$ cells $\left(\mathrm{CD} 11 \mathrm{c}^{+} \mathrm{CD} 206\right)$ cultured without LPS stimulation, the CD11c receptor mean fluorescence intensity (MFI) was lower in patients with IBS than in controls (11 $540.0 \pm 537.5$ vs $13860.0 \pm 893.7, P=0.038$ ) (Fig. 3). In addition, upon analyzing the cultures stimulated with LPS, CD11c expression decreased both in IBS and controls with no significant differences between them (Fig. 3). Moreover, without LPS stimulation the percentage of intermediate cells $\left(\mathrm{CD} 11 \mathrm{c}^{+} \mathrm{CD} 206^{+} \mathrm{CX} 3 \mathrm{CR} 1^{+}\right)$was higher in IBS than controls $(9.5 \pm 1.5 \%$ vs $4.9 \pm 1.4 \% ; P=0.022)$. Such a clear difference was not observed when studying monocytes stimulated with LPS (Fig. 4). Finally, in relation to fractalkine receptor (CX3CR1) expression on M2-Advanced cells, when the difference between with and without LPS stimulation was calculated, IBS showed a slightly decrease in CX3CR1 while there was an increase in controls an increase $(P<0.001)$ (Fig. 5).

\section{Discussion}

Even though the model of monocyte/macrophage cultures from peripheral blood that we used in the current study does not

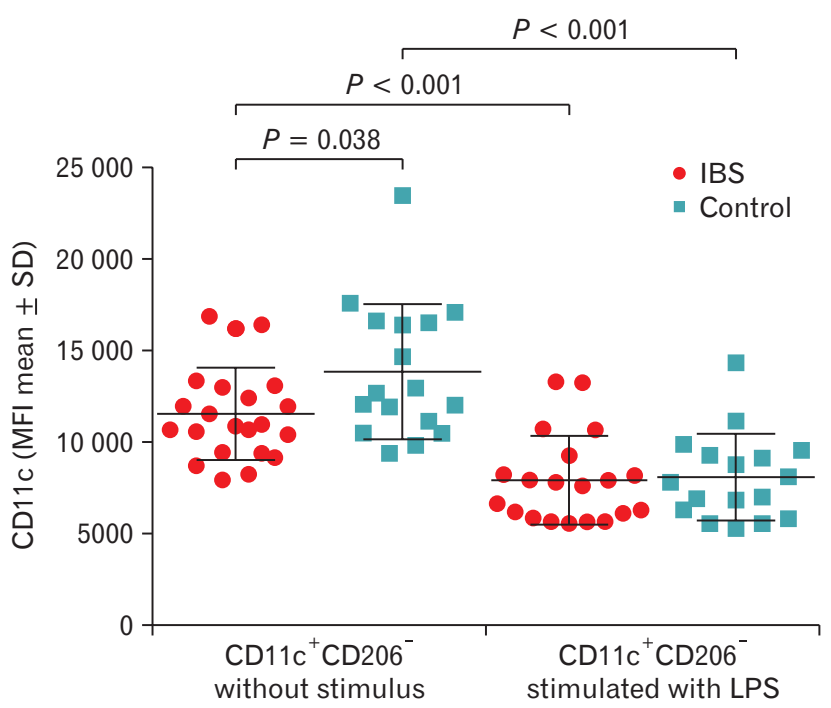

Figure 3. $\mathrm{CD} 11 \mathrm{c}$ expression in $\mathrm{CD} 11 \mathrm{c}^{+} \mathrm{CD} 206^{-}$monocytes, in cultures with and without lipopolysaccharide (LPS) stimulation from irritable bowel syndrome (IBS) patients and controls. The monocytes/ macrophages that were not stimulated in early maturation states, presented a higher level of CD11c mean fluorescence intensity (MFI) expression in controls compared to patients with IBS. There were no differences in cultures with LPS stimulation. 


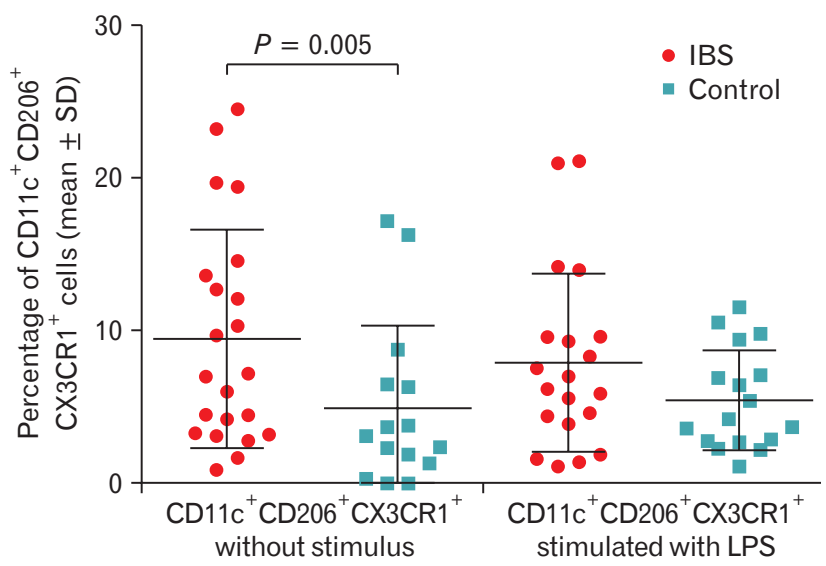

Figure 4. Percentage of $\mathrm{CD} 11 \mathrm{c}^{+} \mathrm{CD} 206^{+} \mathrm{CX} 3 \mathrm{CR} 1^{+}$monocytes, in cultures with and without lipopolysaccharide (LPS) stimulation from irritable bowel syndrome (IBS) patients and controls. In cultures that are not stimulated with LPS from patients with IBS, there is a greater frequency (\%) of monocytes/macrophages that express the different membrane receptors, compared with the controls. No statistically significant difference was found between the groups in cultures with LPS.

exactly replicate the conditions of a response given by macrophages living in the lamina propria of the bowel, our study is based on the fact that the lamina propria macrophages are composed of monocytes recruited from the peripheral blood ${ }^{27}$ that enter the intestine mainly through the TGF- $\beta$ and IL-8 produced by intestinal epithelial cells. ${ }^{28}$ Thus, analyzing a model of MNCs stimulated with LPS is a logical focus for speculating the possible response of these cells to bacterial stimuli and their comparison between IBS patients and controls.

Likewise, it is important to point out that in the 2 studies using a murine model, Bain et $\mathrm{al}^{29}$ and Tamoutounour et $\mathrm{al}^{16}$ proposed that the conditions of the intestine lead to the development of the macrophage in a cascade-like fashion. This is a linear concept in which complete macrophage development up to a tolerant state is possible in a normal lamina propria, whereas an inflammatory state mainly promotes the maturation of proinflammatory response macrophages. This condition suggests that when the monocytes/macrophages are stimulated, as was carried out in our study, it could be possible to observe whether the process of cell development varies between IBS patients and controls, within the logic of a stimulated environment and maturation cascade. Based on the above, the maturation process registered in the present study can be summarized as follows: (1) the presence of LPS induces monocyte/macrophage maturation compared with the cultures with no stimulation, as suggested by less CD11c expression in CD14+ cells in the presence

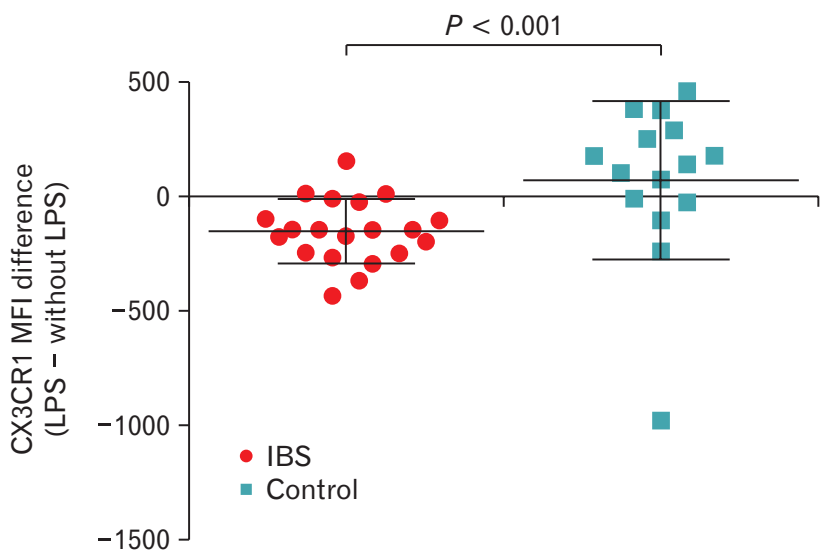

Figure 5. Difference in the fractalkine receptor CX3CR1 expression, with vs without lipopolysaccharide (LPS) stimulation, in irritable bowel syndrome (IBS) patients and controls. The relation between fractalkine receptor expression in cultures with LPS compared to those without LPS (LPS - without LPS), suggests that it is increased in the controls (thus the mean fluorescence intensity: mean fluorescence intensity (MFI) has a positive value), whereas the expression is not increased (MFI is negative or near zero) the cells of patients with IBS.

of LPS in both IBS and controls; (2) a more activated basal state is suggested in the monocytes/macrophages of patients with IBS with respect to healthy controls, based on the lower expression of CD11c in M1 cells in cultures without LPS stimulation in IBS vs controls; and (3) a faster development to an immune regulatory state is observed in the controls compared with the IBS patients, as suggested by the presence of an increased expression of the fractalkine receptor (CX3CR1) in response to LPS only in controls but not in the IBS (Fig. 1).

Therefore, in line with the proposed cascade fashion development, it could be speculated that the presence of the different membrane markers found in the current study, suggests that there is a difference between patients with IBS and the controls in the monocyte/macrophage maturation process. In this sense, the CD11c, ${ }^{17}$ $\mathrm{CD}_{206}{ }^{29}$ and $\mathrm{CX} 3 \mathrm{CR}^{17}$ markers are not expressed in peripheral monocytes, but rather in a state of residence in the bowel, ${ }^{17}$ which in our study is very likely associated with the cell-culture conditions. In the bowel, the macrophages express the $\mathrm{CD} 11 \mathrm{c}^{17}$ marker, although its presence is associated with differentiated macrophages. In general, it is not found in the cells with the M2-like phenotype, whose main function is the regulation of inflammation ${ }^{30}$ mediated by $\mathrm{T}$ cells. ${ }^{31}$ That is to say, as the macrophage matures, there is an increase expression of CD11c, and as the cell continues to develop, the CD11c expression declines until it completely disappears. Like- 
wise, CD206 and CX3CR1 marker expression is increased as the cells mature in the bowel and they are equally associated with the M2-like phenotype, which can involve metastable state functions with the dynamics of the microbiota, as well as a constant remodeling of the intestinal tissue. ${ }^{32}$

In accordance with all of the above, it is to be expected that the $\mathrm{CD} 206^{+} \mathrm{CX} 3 \mathrm{CR} 1^{+}$subpopulation (in relation to the level of cellular activation and percentage) is the one that presents with a greater state of development, whereas $\mathrm{CD} 206^{-} \mathrm{CX} 3 \mathrm{CR}^{-}{ }^{-}$is the less advanced. Therefore, the general observation of the reduced expression of CD11c in the cells cultured with LPS in our study suggests a maturation process in the presence of a bacterial stimulus, as is to be expected in the cascade proposal of monocyte development. ${ }^{17}$ Likewise, the significantly higher MFI of CD11c in M1 control cells cultured without LPS stimulation suggests that, under basal conditions, the cells of the controls are less developed than those from patients with IBS.

On the other hand, despite the fact that in the M2 subpopulation the level of CX3CR1 receptor expression is slightly higher in the cells stimulated with LPS from patients with IBS compared with the controls, upon subtracting the CX3CR1 receptor expression of the $\mathrm{CD} 11 \mathrm{c}^{-} \mathrm{CD} 206^{+}$cells stimulated with LPS from the non-stimulated cells, a reduction in CX3CR1 expression in IBS is suggested, which is contrary to that observed in the controls. This is concordant with the previous hypothesis that there are different initial states of maturation between IBS and controls and that they become more alike after LPS stimulation. There is most likely a faster response in the controls toward a tolerance state in the presence of the TLR ligand, LPS, despite being "behind" in the state with no stimulation. In other words, even though the data suggest that in the absence of bacterial stimulus the patients with IBS can present with a superior activation state, in the presence of LPS it becomes balanced or even reduced, compared with that which is observed in the controls.

In summary, our results suggest that the monocytes/macrophages of the patients with IBS have a previous activation state, but then after stimulation with TLR bacterial ligands, such as LPS from $E$. coli, this difference diminishes when compared with the controls. This loss of difference in the maturation state between patients with IBS and controls may be related to an immunologic activation by pathogen-associated molecular pattern (PAMP)-type ligands, that are capable of modulating this immune activation (as has recently been proposed in macrophages from IBS patients). ${ }^{14}$ In this sense we could speculate that TLR modulation by PAMP ligands could be related to the mechanisms by which probiotics ${ }^{33}$ and luminal antibiotic use ${ }^{34}$ produce improvement in IBS symptoms. It must be kept in mind that this monocyte modulation can, in turn, be related to the cytokines produced by lymphocytes in co-cultures.

Our study has some limitations. First, we diagnosed IBS based on the Rome II criteria and not Rome III or the newer Rome IV criteria. However, in Mexico we have found that the Rome III criteria lack sensitivity for detecting IBS and the majority of cases are distributed in unsubtyped IBS according to those criteria. ${ }^{35}$ In addition, the recently released Rome IV criteria have not yet been translated into Spanish but we need to wait and see they are more adequate for our population. Second, we did not study patients with PI-IBS, which represent $5 \%$, a very small group, in our population. ${ }^{36}$ Nevertheless, this can turn out to be more of a strength, because our results suggest that even without a history of infectious enteritis for the development of IBS, the patients present with an alteration in immune regulation in the presence of bacterial pathogens or it could be a response to an alteration in the gut microbiota. Third, we did not analyze the basal expression of the maturation markers in non-cultured monocytes/macrophages, which should be studied. Finally, we must reproduce our experiment with intestinal mucosal monocytes/macrophages to confirm that what we found in the peripheral blood cells reflects what occurs in the bowel.

In conclusion, monocyte/macrophage, maturation appears to be more advanced in patients with IBS compared to controls in cultures without stimulation. However, the contact with PAMPs, such as $E$. coli-derived LPS, generated a greater maturation process in the monocytes/macrophages in the controls, but not in IBS. This last result suggests an alteration in immune regulation in patients with IBS.

Financial support: This study was financed by the PAPIIT IN210010 project of the Research and Technologic Innovation Project Support Program (Programa de Apoyo a Proyectos de Investigación e Innovación Tecnológica), DGAPA-Universidad Nacional Autónoma de México (UNAM). Oscar Rodríguez-Fandiño received a grant from the Consejo Nacional de Ciencia y Tecnología (CONACyT) (No. 336205), as a doctoral student in Biomedical Sciences at the Faculty of Medicine of the Universidad Nacional Autónoma de México (UNAM).

Conflicts of interest: Max Schmulson has received grant supports from Alfa Wassermann, Nestle Ltd, and Nycomed/Takeda Mexico. He has served on the Advisory Board of Alfa Wassermann and has been a consultant for Almirall, Commonwealth Laboratories Inc, Commonwealth Diagnostics International Inc, 
Janssen, Nestle Ltd, Novartis, Procter and Gamble, Senosiain, and Takeda Mexico. He has also been a speaker for Alfa Wassermann, Janssen, Mayoli-Spindler, and Takeda Mexico. Joselín HernándezRuiz has received grant support from Alfa Wassermann. Yolanda López-Vidal has been a consultant for Alfa Wassermann. Oscar Rodríguez-Fandiño, Luis Charua-Guindic, and Galileo Escobedo have nothing to declare.

Author contributions: Oscar Rodríguez-Fandiño: patient selection and enrollment, sample collection, and processing, analyzing, and interpreting the data and drafting the manuscript; Joselín Hernández-Ruíz: planning and study conceptualization, flow cytometry analyses, data analyses and interpretation; Yolanda LópezVidal: analyzing and interpreting the data; Luis Charúa: patient selection and enrollment, sample collection; Galileo Escobedo: data analysis and interpretation; and Max J Schmulson: study planning, conceptualization and funding, patient selection, analyzing and interpreting the data, and drafting the manuscript. All authors have approved the final draft.

\section{References}

1. Dunlop SP, Jenkins D, Neal KR, Spiller RC. Relative importance of enterochromaffin cell hyperplasia, anxiety, and depression in postinfectious IBS. Gastroenterology 2003;125:1651-1659.

2. Quigley EM. Antibiotics for irritable bowel syndrome: hitting the target, but what is it? Gastroenterology 2011;141:391-393.

3. Salonen A, de Vos WM, Palva A. Gastrointestinal microbiota in irritable bowel syndrome: present state and perspectives. Microbiology 2010;156(Pt 11):3205-3215.

4. Hyland NP, Quigley EM, Brint E. Microbiota-host interactions in irritable bowel syndrome: epithelial barrier, immune regulation and braingut interactions. World J Gastroenterol 2014;20:8859-8866.

5. Rodríguez-Fandiño O, Hernández-Ruiz J, Schmulson M. From cytokines to toll-like receptors and beyond - current knowledge and future research needs in irritable bowel syndrome. J Neurogastroenterol Motil 2010;16:363-373.

6. Ohman L, Simrén M. Pathogenesis of IBS: role of inflammation, immunity and neuroimmune interactions. Nat Rev Gastroenterol Hepatol 2010;7:163-173

7. Lee K, Tack J. Altered intestinal microbiota in irritable bowel syndrome. Neurogastroenterol Motil 2010;22:493-498.

8. Maxwell P, Rink E, Kumar D, Mendall MA. Antibiotics increase functional abdominal symptoms. Am J Gastroenterol 2002;97:104-108.

9. Ford AC, Quigley EM, Lacy BE, et al. Efficacy of prebiotics, probiotics, and synbiotics in irritable bowel syndrome and chronic idiopathic constipation: systematic review and meta-analysis. Am J Gastroenterol 2014;109:1547-1561.

10. Moayyedi P, Ford AC, Talley N, et al. The efficacy of probiotics in the treatment of irritable bowel syndrome: a systematic review. Gut 2010;59:325-332.

11. Zhou Q, Zhang B, Verne GN. Intestinal membrane permeability and hypersensitivity in the irritable bowel syndrome. Pain 2009;146:41-46.

12. Piche T, Barbara G, Aubert P, et al. Impaired intestinal barrier integrity in the colon of patients with irritable bowel syndrome: involvement of soluble mediators. Gut 2009;58:196-201.

13. Bashashati M, Rezaei N, Shafieyoun A, et al. Cytokine imbalance in irritable bowel syndrome: a systematic review and meta-analysis. Neurogastroenterol Motil 2014;26:1036-1048.

14. Ohman L, Lindmark AC, Isaksson S, et al. Increased TLR2 expression on blood monocytes in irritable bowel syndrome patients. Eur J Gastroenterol Hepatol 2012;24:398-405.

15. Bain CC, Bravo-Blas A, Scott CL, et al. Constant replenishment from circulating monocytes maintains the macrophage pool in the intestine of adult mice. Nat Immunol 2014;15:929-937.

16. Tamoutounour S, Henri S, Lelouard H, et al. CD64 distinguishes macrophages from dendritic cells in the gut and reveals the Th1-inducing role of mesenteric lymph node macrophages during colitis. Eur J Immunol 2012;42:3150-3166.

17. De Calisto J, Villablanca EJ, Mora JR. FcyRI (CD64): an identity card for intestinal macrophages. Eur J Immunol 2012;42:3136-3140.

18. Rodríguez-Fandiño O, Hernández-Ruíz J, López-Vidal Y, et al. Intestinal recruiting and activation profiles in peripheral blood mononuclear cells in response to pathogen-associated molecular patterns stimulation in patients with IBS. Neurogastroenterol Motil 2013;25:872, e699.

19. López-Colombo A ${ }^{1}$, Morgan D, Bravo-González D, Montiel-Jarquín A, Méndez-Martínez S, Schmulson M. The epidemiology of functional gastrointestinal disorders in Mexico: a population-based study. Gastroenterol Res Pract 2012;2012:606174.

20. Schmulson M, Ortiz O, Santiago-Lomeli M, et al. Frequency of functional bowel disorders among healthy volunteers in Mexico City. Dig Dis 2006;24:342-347.

21. Thompson W, Longstreth G, Drossman DA, et al. Functional bowel disorders and functional abdominal pain. In: Drossman DA, Corazziari E, Talley NJ, Thompson WG, Whitehead WE, eds. Rome II: The functional gastrointestinal disorders. 2nd ed. McClean, VA: Degnon Associates Inc. 2000.

22. Dunlop SP, Jenkins D, Spiller RC. Distinctive clinical, psychological, and histological features of postinfective irritable bowel syndrome. Am J Gastroenterol 2003;98:1578-1583.

23. Godoy-Ramirez K, Franck K, Mahdavifar S, Andersson L, Gaines H. Optimum culture conditions for specific and nonspecific activation of whole blood and PBMC for intracellular cytokine assessment by flow cytometry. J Immunol Methods 2004;292:1-15.

24. McKernan DP, Gaszner G, Quigley EM, Cryan JF, Dinan TG. Altered peripheral toll-like receptor responses in the irritable bowel syndrome. Aliment Pharmacol Ther 2011;33:1045-1052.

25. Germann A, Schulz JC, Kemp-Kamke B, Zimmermann H, von Briesen H. Standardized serum-free cryomedia maintain peripheral blood mononuclear cell viability, recovery, and antigen-specific T-cell response compared to ftal calf serum-based medium. Biopreserv Biobank 2011;9:229- 
236.

26. Bain CC, Mowat AM. Macrophages in intestinal homeostasis and inflammation. Immunol Rev 2014;260:102-117.

27. Smith PD, Smythies LE, Shen R, Greenwell-Wild T, Gliozzi M, Wahl $\mathrm{SM}$. Intestinal macrophages and response to microbial encroachment. Mucosal Immunol 2011;4:31-42.

28. Smythies LE, Maheshwari A, Clements R, et al. Mucosal IL-8 and TGF-beta recruit blood monocytes: evidence for cross-talk between the lamina propria stroma and myeloid cells. J Leukoc Biol 2006;80:492499.

29. Bain CC, Scott CL, Uronen-Hansson H, et al. Resident and proinflammatory macrophages in the colon represent alternative contextdependent fates of the same Ly6C(hi) monocyte precursors. Mucosal Immunol 2012;6:498-510.

30. Hunter MM, Wang A, Parhar KS, et al. In vitro-derived alternatively activated macrophages reduce colonic inflammation in mice. Gastroenterology 2010;138:1395-1405.
31. Sica A, Mantovani A. Macrophage plasticity and polarization: in vivo veritas. J Clin Invest 2012;122:787-795.

32. Zigmond E, Jung S. Intestinal macrophages: well educated exceptions from the rule. Trends Immunol 2013;34:162-168.

33. Barbara G, Zecchi L, Barbaro R, et al. Mucosal permeability and immune activation as potential therapeutic targets of probiotics in irritable bowel syndrome. J Clin Gastroenterol 2012;46(suppl):S52-S55.

34. Saadi M, McCallum RW. Rifaximin in irritable bowel syndrome: rationale, evidence and clinical use. Ther Adv Chronic Dis 2013;4:71-75.

35. Schmulson M, Lopez-Colombo A, Mendoza-Gómez A, MontielJarquin A, Morgan D. The Rome III adult questionnaire in SpanishMexico has a low sensitivity for identifying IBS and higher sensitivity for uninvestigated dyspepsia. Gastroenterology 2012;142:S829-S830.

36. Ortiz O, Remes-Troche J, Valdovinos M, et al. [Frequency of extero and interoceptive factors triggering the first manifestation of irritable bowel syndrome (IBS) in a third level hospital in Mexico.] [abstract] Rev Gastroenterol Mex 2003;68:98-99. [Spanish] 